\title{
Acoustic emission based prediction of local stress exposure
}

\author{
MARKUS G. R. SAUSE ${ }^{1}$, STEFAN SCHMITT ${ }^{1}$, BIRTE HOECK ${ }^{2}$, ANDREAS MONDEN ${ }^{1}$ \\ 'University of Augsburg, Institute for Materials Resource Management, Mechanical Engineering, \\ D-86135 Augsburg \\ ${ }^{2}$ MT Aerospace AG, Franz-Josef-Strauß-Str. 5, D-86153 Augsburg
}

\section{Introduction}

Structures made from fiber-reinforced materials are designed for excellent strength-toweight and stiffness-to-weight ratios. However, a skilled lightweight design using these materials requires reliable tools to aid the structural analysis. For structural analysis, there are failure theories enabling the prediction of failure initiation and the related degradation of the material. Today, a multitude of theoretical concepts exists in order to aid the engineer to predict the occurrence of failure, yet none of them outperforms the other in a general load situation [1-3]. In industrial applications, the uncertainty caused in the design process of composite structures results in a huge number of tests to be completed ranging from small-scale specimens to large-scale structures in order to achieve a level of confidence in the particular material and the particular design. Especially for space applications, full-scale tests for structures with dimensions of several meters are extremely time and cost consuming. Therefore, a dedicated scaling philosophy needs to be established to perform sub-scale tests and to transfer the results from sub-scale components to full-scale.

One method that is flexible enough to assist in the interpretation of failure on smallscale and large-scale is acoustic emission (AE) analysis. During rapid internal displacements, such as during crack initiation and growth, elastic stress-waves are released. These propagate in the ultrasonic range and can be detected by sensitive piezoelectric sensing systems. For the specific implications of measurements on fiberreinforced materials a comprehensive introduction for this topic is found in $[4,5]$. 
In typical thin-walled fiber-reinforced composites, AE signals can be detected at a significant distance from source position, however, they experience attenuation effects and guided wave propagation. There is no strict upper limit for the distance of propagation, but based on the strength of the source it is likely that sensing systems might fall below the detection threshold of the system. This concept is known as probability of detection (PoD) in nondestructive testing and has been implemented for AE analysis in [6], following earlier considerations by Pollock [7]. However, this is solely useful to ensure detection of $A E$ signals but does not deal with the falsification of wave information during propagation. As pointed out in $[8,9]$ and described in test standards such as DIN EN 15857, AE signal features will significantly be affected by their distance of propagation. Accordingly, to compensate these effects one can inversely calculate the feature values of the wave at the source position, if the latter and the propagation effects are known. A rigid proposal to implement such strategies has been made by [8] and is followed in this study. Prerequisite to this approach is the knowledge of the (xyz)source coordinate corresponding to the detected signals. This information is obtained by source localization algorithms, which typically require knowledge of sound velocities in the structure and are limited to very simple geometries (plates, cubes, spheres, cylinders, ...) [10]. In order to approach a more complex geometry, recent studies proposed the use of artificial neural networks [11] or mapping techniques [12,13] for this task.

Specifically for large-scale structures, testing costs can be significant and prediction of ultimate loads obtained without ultimately destroying the test item offer huge potential. Similar, for applications in quality control the forward prediction of ultimate loads based on information obtained during proof testing would offer a valuable contribution to product safety.

Several concepts have been used to perform AE based prediction of failure loads, many of them resulting from the need to predict burst pressure levels of fiber-reinforced vessels. The usual load schedule applied (according to e.g. ASTM E 1067) is a cyclic load / unload scheme as seen in Figure 1. The earliest application of this schedule in 
combination with AE analysis goes back to Kaiser in 1950 [15]. He demonstrated for the case of metallic materials, that the AE usually re-initiates after exceeding the previously reached load level. This behavior of materials has been termed the Kaisereffect to honor his pioneering work in the field of AE. However, for fiber-reinforced materials the AE signals often initiate at lower load levels than previously achieved. This fundamental observation goes back to the work of Fowler [16]. Since this first publication in 1977, the evaluation of the Felicity ratio became an important concept to understand the progression of failure in fiber-reinforced materials using $\mathrm{AE}$.

As a similar measure for damage progression in composite materials, the Shelby ratio has been proposed by Downs and Hamstad [14]. It is different from the Felicity ratio in that the AE evaluation is based on the hits detected during un-loading of the test item. When reaching a certain evaluation criterion (such as a total number of accumulated hits) during the un-loading phase the corresponding load level is used for the evaluation. This load level is related to the previously reached maximum load. This approach has been used to assess the quality of composite pressure vessels since those with prior damage generate noticeable amounts of $\mathrm{AE}$ during de-pressurization [14].

Other related quantities, which can be derived from $A E$ measurements, are energybased ratios. As recently introduced in [15], these are based on the accumulated energies reached in a cycle in relation to the energy reached in the previous cycles. The corresponding onset values are related to the previously reached loads (see section 2.1 for definition). Similar to the Felicity ratio and the Shelby ratio, these can be defined independent of the test component size and the AE activity as has been indicated in [15] and will be demonstrated in the following work.

In the past, Felicity ratio and Shelby ratio were correlated with the burst pressure of fiber-reinforced composite vessels and indicated that their values are highly correlated [14,16-18]. Based on this observation Waller et al. extended this approach into a forward prediction routine, collecting the Felicity ratio for a certain number of cycles until failure [19]. They proposed to establish a critical Felicity ratio as material property 
and evaluated their concept for fiber-reinforced strands and pressure vessels, which was conceptually extended by the approach presented in [15].

Another independent approach to predict the ultimate load of fiber-reinforced composite vessels was proposed by Hill and Walker using the recorded AE amplitude distributions as input data [20-22]. They used artificial neural networks to classify the $\mathrm{AE}$ data and based their forward prediction on a second supervised training stage on the entirety of the $A E$ dataset. This provided evidence that artificial neural networks are flexible enough to perform such a predictive task. A similar approach has also been applied by other groups for prediction of tensile coupon strength [23].

Within this study, the application of failure prediction techniques to large composite pressure vessels is demonstrated. Therefore, the previous approach in [15] is extended to enable calculation of the local stress exposure (load ratio). The small-scale and large-scale pressure vessels are used to demonstrate the combination of PoD studies, neural network based source localization and test evaluation. For the latter, the focus is on the prediction of the burst pressure as well as the evaluation and discussion of local load ratios.

\section{Failure prediction concept}

Recently, an extension of the concepts proposed by Waller et al. [19] and Hill and Walker [20-22] to predict composite failure was proposed [15]. Accordingly, the global approach is only briefly introduced herein and the interested reader is referred to [15] for a more detailed presentation.

For this work, a supervised classification scheme applying artificial neural networks was applied. The first stage is the generation of a test database to establish the relationship between $A E$ parameters and load levels. The second stage is the application of the established relationship to a new test item to perform a forward prediction.

For the first stage, the required steps are: 
1. Definition of $A E$ parameters to evaluate in each cycle

2. Evaluation of these parameters during load/unload cycles until failure

3. Establishing a relationship between AE parameters and ratio of load-to-failure For the second stage, the steps are slightly different:

1. Definition of $A E$ parameters to evaluate in each cycle

2. Evaluation of these parameters during load/unload cycles until a certain load level

3. Using established relationship to predict ratio of load-to-failure in each cycle

4. Extrapolating the load-to-failure ratio to unity thereby predicting the global failure level and adding an estimation of prediction error

\subsection{Global failure prediction}

In this study a step-wise cyclic loading until failure is applied. The test specimen is subject to a monotonic increasing load until a certain load level $L_{1}$ is reached. Then the specimen is partially unloaded (Figure 1-b). In the next "cycle" the load is increased again until a certain load level $L_{2}$ is reached, which exceeds the previously reached load level. This process is repeated $\mathrm{N}$-times up to a certain maximum load level $L_{\max }$ or up to specimen failure $L_{\text {failure }}$. This gives rise to the definition of the load ratio $L R$ for cycle $N$ :

$L R=L_{N} / L_{\text {failure }}$

This global load ratio is identical to the global stress exposure of the structure (confirm Puck's failure theory $[24,25])$, which is also known as reserve factor, when using $L R^{-1}$ instead.

During loading, $A E$ signals are acquired and can be used to calculate the following $A E$ criteria. Based on the definition in [15] five different Felicity ratios and three energetic criteria for each cycle $N$ are calculated. The general definition of the Felicity ratio is using the onset of $\mathrm{AE}$ hits in the present cycle $L_{F R, N}$ and relating this value to the previously reached load $L_{N-1}$ :

$F R_{N}=L_{F R, N} / L_{N-1}$ 
In this case, the onset values $L_{F R, N}$ of the Felicity ratios are defined as relative shares for fractions $5 \%, 10 \%, 15 \%$ and $20 \%$ of the total number of hits during the ascending part of the cycle. This is denominated FR5, FR10, FR15 and FR20 in the following. In addition, the average Felicity ratio is defined as the arithmetic mean of these four values:

$\langle F R\rangle=\frac{1}{4}(F R 5+F R 10+F R 15+F R 20)$

For the energetic values, the features "signal amplitude", "signal energy" and "averagesignal-level" are considered. The onset is defined as load value $L_{E, N}$ where their previously reached accumulated value $A_{N-1}$ is exceeded, i.e. at the value $A_{N} \geq$ $2 \cdot A_{N-1}$. This results in the definition:

$E R_{N}=L_{E, N} / L_{N-1}$

In the first stage, a symbolic relationship $f_{\text {opt }}$ between $F R_{N}$ and $E R_{N}$ values and the applied $L R$ is established. Based on the detailed description in [15], this is achieved by means of a backpropagation neural network and is not repeated in detail here.

In the second stage, this established relationship $f_{o p t}$ can be applied to predict unknown $L R$ values:

$L R_{N}=f_{\text {opt }}\left(F R_{N}, E R_{N}, \ldots\right)$

This allows predicting the load ratio of cycle $N$ based on $F R_{N}$ and $E R_{N}$ values. For the forward prediction of the global failure load, several $L R$ values are required. Based on the original proposal by Waller et al. [19], this can be used for a linear extrapolation algorithm. Intersection of the extrapolation line with $L R=1$ is then used to estimate the global failure load. In the following we apply the specific implementation presented in [15], with examples shown for the test cases in Section 4.1 and 4.2.

\subsection{Local failure prediction}

The description of Section 2.1 assumes the use of all AE signals for the calculation of $L R$ values. However, for larger test objects it is possible to refine this global approach to a local calculation of $L R$ values. In case of localized AE sources, it is possible to segment the global $A E$ dataset based on the (xyz)-position. As seen in Figure 1, a simple volumetric binning approach is used. The volume enclosing the test structure under investigation is divided in sub-volumes of identical size. With reference to the 
coordinate system of Figure 1, this is making use of $i$ sub-divisions along the $x$-axis, $j$ sub-divisions along the $\mathrm{y}$-axis and $k$ sub-divisions along the z-axis. For the given geometry, an adapted (cylindrical, spherical, ...) segmentation could be selected as well. However, the sub-division based on the Cartesian coordinates appeared as the generic choice and is applicable to geometries other than pressure vessels. Limiting the $\mathrm{AE}$ signals to a subset with source coordinates falling within the sub-volume $i j k$ allows applying equation (5) locally:

$L R_{N, i j k}=f_{\text {opt }}\left(F R_{N, i j k}, E R_{N, i j k}, \ldots\right)$

Applying equation (6) for each cycle and each sub-volume $x=1, \ldots, i, y=1, \ldots, j$ and $z=1, \ldots, k$ as seen in Figure 1 allows calculating spatially resolved values of $L R$ for each cycle. The size of each sub-volume is chosen based on a measure of the source location precision, i.e. the uncertainty of the source location is taken as value for the dimension of the segmentation seen in Figure 1.

(a)

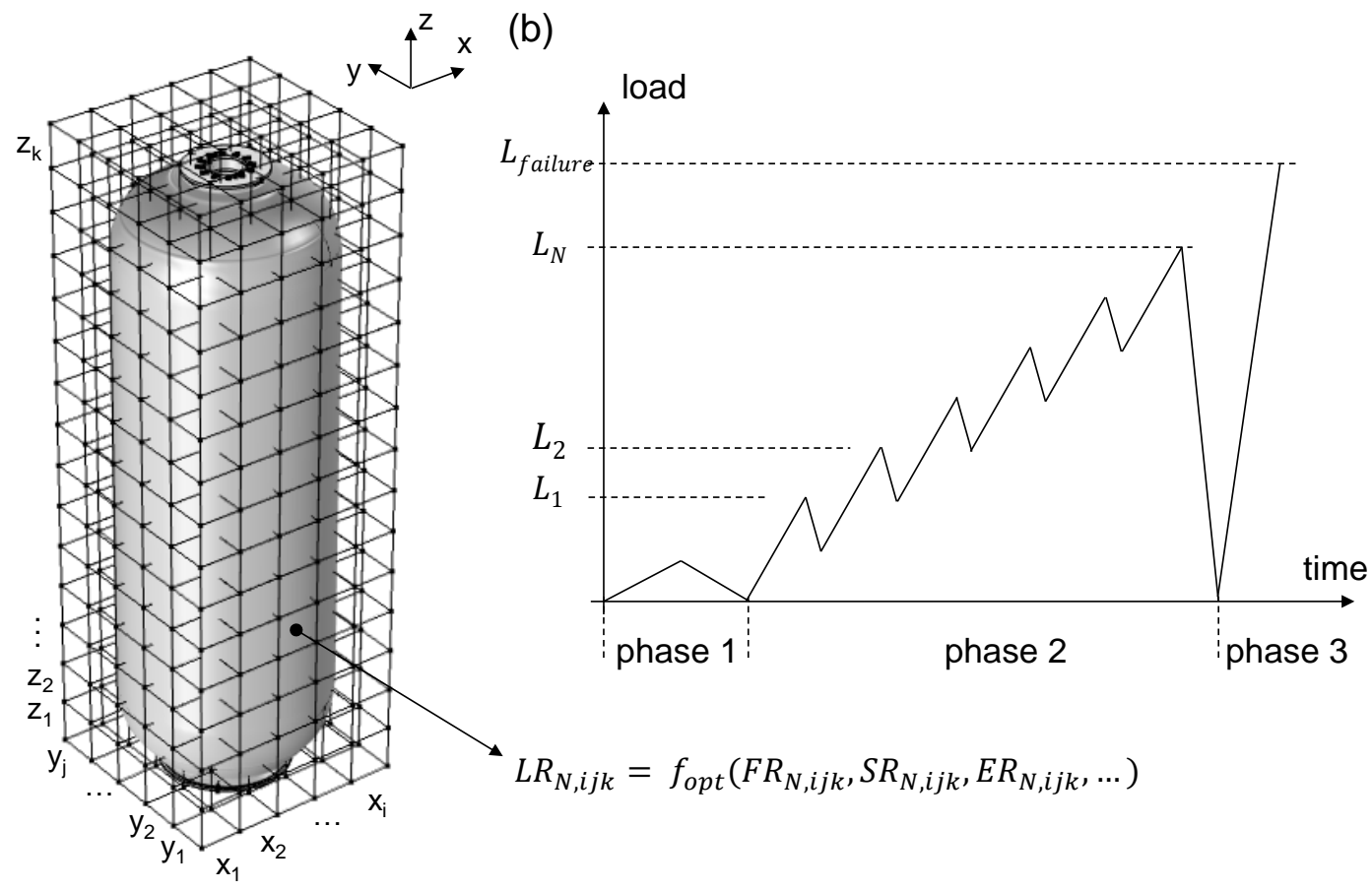

Figure 1: Scheme for segmentation of a 3D-object into local domains for failure prediction (a) and load schedule used in this test to obtain cyclic test data (b). 


\section{Experimental}

In the following, the experimental setups for the two different sized classes of pressure vessels in this study are presented. In order to apply the failure prediction approach described above, a reference database is used, which is described first.

\subsection{Reference database}

In order to apply the neural network based failure prediction established in [15], a training stage is required to establish a symbolic relationship $f_{\text {opt }}$ between cyclic $\mathrm{AE}$ criteria and the applied load ratio (see Section 2.1). Since the pressure vessels are manufactured using dry filament winding and dry fiber placement processes with intermediate modulus (IM) fibers, the test plates are fabricated accordingly. For the subsequent resin infusion process an appropriate epoxy resin system has been chosen and was cured following the material supplier's recommendation. For the reference database, a significant number of lab scale tests has been carried out, whose detailed description is beyond the scope of this article. Following the principal outline in [15] all specimens were tested in cyclic loading to establish a mixed database, following the principles of the test standards and settings listed in Table 1. The test conditions are selected to represent the relevant failure modes expected on structural scale, which are tensile failure, delamination failure, local buckling (bending failure) and adhesive bonding failure (for LPV-2 test with skirt region only, confirm Section 3.2). The 4-point bending test is applied on specimens directly cut from untested large-scale pressure vessels. Accordingly, these incorporate the full complexity of the layup due to the filament winding process. Load increments were defined accordingly to reach 10 to 20 cycles before ultimate failure (i.e. rupture for the tensile test and lap shear cases and exceedance of the maximum strength for the double cantilever beam, end notched flexure and bending cases).

All test data is acquired using identical AE settings. Therefore, the same measurement chain consisting of WD type multi-resonant AE sensors (Mistras), a medium viscosity silicone grease type Korasilone (Bayer) as couplant, 2/4/6 preamplifiers (Mistras) at $40 \mathrm{~dB}_{\mathrm{AE}}$ gain and $35 \mathrm{~dB}_{\mathrm{AE}}$ detection threshold were used. The trigger settings are $10 / 80 / 300 \mu \mathrm{s}$

(Peak-Definition-Time/Hit-Definition-Time/Hit-Lockout-Time). Bandwidth limitation is given by a $20 \mathrm{kHz}-1200 \mathrm{kHz}$ bandpass integrated in the 
preamplifier and an additional $20 \mathrm{kHz}-1000 \mathrm{kHz} 6^{\text {th }}$ order analogue Butterworth filter installed on the type PCl-2 acquisition cards (Mistras). Both are selected in accordance with the bandwidth of the WD sensor type ranging from $125 \mathrm{kHz}$ to $1000 \mathrm{kHz}$. For all tests of the reference database, WD sensors are attached using suitable clamp systems. To ensure all relevant AE signals are detected, for all reference database tests we evaluated the PoD following [6], assuming the extreme case of measured attenuation of $\alpha=57.5 \mathrm{~dB} / \mathrm{m}$ in the frequency range up to $1 \mathrm{MHz}$. For the relatively short distances of signal propagation, this results in PoD $\geq 99 \%$ for all cases. Accordingly, this data may also be used as PoD reference in the following. For this approach, all test cases of table 1 are mixed with equal weight to obtain a reference signal amplitude distribution (see [6] for a detailed description).

Based on the recorded $\mathrm{AE}$ criteria and measured load ratios, an artificial neural network is trained to yield the function $f_{\text {opt }}$. This is implemented as multi-layer feedforward neural network in MATLAB with two hidden layers and twenty neurons to perform the function approximation using a Levenberg-Marquardt algorithm. Applying the global failure prediction routine within the reference database (excluding the test to be predicted) all specimen's failure strength (maximum load) are predicted within the margin of error. This reference database is used in the following to check its applicability for prediction of structural components.

Table 1: Summary of test type, sample statistics, mechanical test standards and AE settings to obtain reference database.

\begin{tabular}{|l|c|c|c|}
\hline Test type & $\begin{array}{c}\text { Samples } \\
\text { tested }\end{array}$ & Test standard & AE instrumentation \\
\hline Tensile tests & 20 & $\begin{array}{c}\text { DIN EN ISO 527-4 / DIN EN } \\
\text { ISO 527-5 }\end{array}$ & 2 channels \\
\hline $\begin{array}{l}\text { Double } \\
\text { cantilever beam }\end{array}$ & 24 & ASTM D 5528 & channels \\
\hline $\begin{array}{l}\text { End notched } \\
\text { flexure }\end{array}$ & 10 & ASTM D 7905 & 4 channels \\
\hline 4-point bending & 6 & $\begin{array}{c}\text { n/a - sample size 405 } \times 33 \\
\times 45 \text { (length } \times \text { height } \times \\
\text { width) mm } m^{3}\end{array}$ & 2 channels \\
\hline Lap-Shear tests & 4 & ASTM D 5868 & \\
\hline
\end{tabular}




\subsection{Small-scale pressure vessels}

As first step, the failure prediction approach is applied to a small pressure vessel of $560 \mathrm{~mm}$ length and $151 \mathrm{~mm}$ diameter, which is two to three times larger than the specimens included in the reference database. All pressure vessels are built from the same material as used for the reference database using dry filament winding with subsequent resin infusion and curing. Due to the filament winding pattern it is expected that the pressure vessels are subject to non-uniform stresses that will differ from the uniform load cases listed in table 1. The three tested vessels are designated SPV-1, SPV-2 and SPV-3.

These pressure vessel are equipped with several strain gages bonded parallel to the fiber direction. At each equator several strain gages are bonded on the circumference to cover this critical position with a complex internal stress state and another measurement plane is located in the middle of the cylindrical range where also several strain gages are bonded to record data from a more uniformly loaded area. Thus, three measurement sections are recorded during the test.

The principal test plan for loading the pressure vessels is shown in Figure 1. In the first phase, a single cycle with minimal pressure is applied to the vessel to calibrate, check mounted strain gages, and to ensure no leakages occur in the pressurization system. In the second phase, a number of step-wise increased pressurization cycles with intermediate partial unloads follows, which is designated cyclic loading in the following. As a final phase, the vessel is visually inspected and subsequently loaded to failure. During all phases, the applied pressure and strain gage values at the designated positions are recorded.

The $A E$ instrumentation is based on a PoD study using attenuation mapping as described in [6]. As seen in Figure 2-a, the selected 11 sensor positions allow a coverage with a PoD $\geq 96 \%$ using an attenuation mapping approach as introduced in [6]. All AE acquisition settings are in accordance with the reference database, except for the trigger settings, which are chosen as 20/80/1500 $\mu$ s (Peak-Definition-Time/HitDefinition-Time/Hit-Lockout-Time) to account for the change in signal length due to guided wave dispersion at larger propagation distances. In this case, all AE sensors are attached using custom-built systems with adhesive bonding of the attachment 
frame to the vessels (see Figure 3). For the attenuation mapping as well as for the neural network based source localization procedure, a training grid of $\geq 135$ (xyz)locations is used. Test signals are acquired using an ASTM E 976 standardized HsuNielsen source. For all AE signals, a simple threshold based arrival time estimation is used, as advanced criteria, such as the Akaike Information Criterion (AIC) are computationally too intense for the $>10^{6}$ hits for the LPV cases. Following the concept proposed in [11] the obtained $\Delta \mathrm{t}$-values are input to the training stage of an artificial neural network with two hidden layers and ten neurons each and are mapped against the corresponding (xyz)-locations. This allows for AE source localization with a calculated accuracy of $\leq 36 \mathrm{~mm}$. For global and local prediction of failure, only localized $A E$ sources are taken into account.

(a)

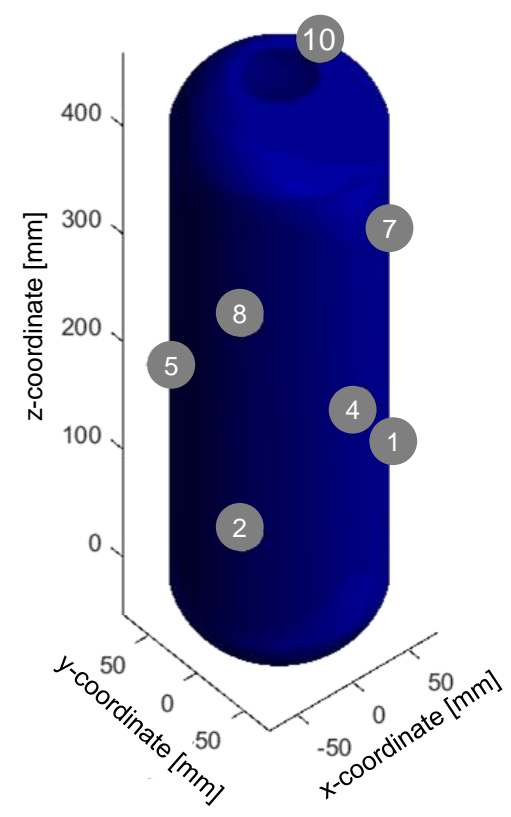

(b)

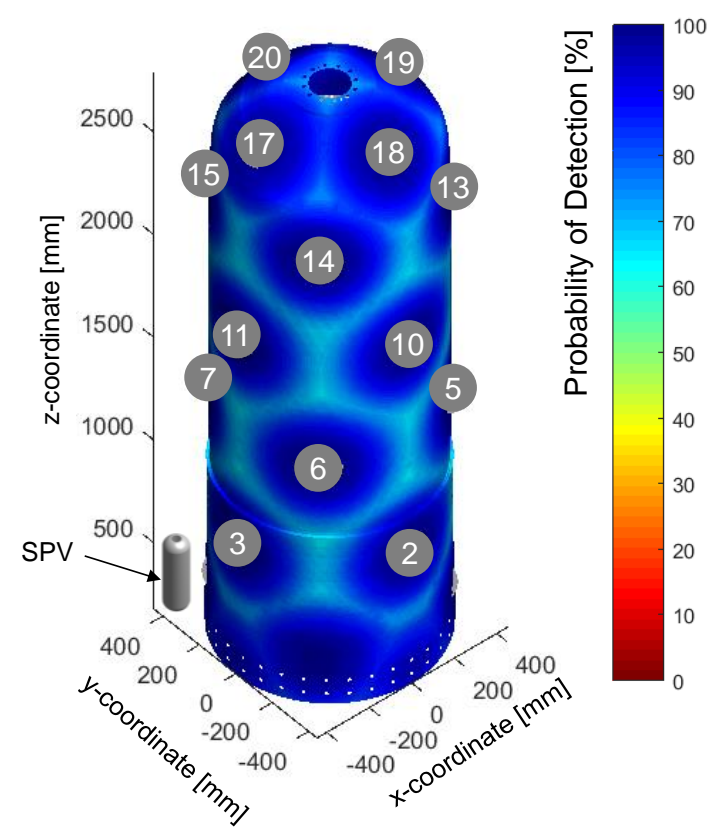

Figure 2: Visualization of sensor positions installed on small pressure vessel SPV-1 (a) and large pressure vessel LPV-2 (b) including color-coded PoD. Some of the sensor locations are hidden by the vessels. For size comparison, the small-scale pressure vessel is additionally scaled and inset on the right to fit with the large pressure vessel.

\subsection{Large-scale pressure vessels}

To demonstrate the scale independence of the proposed failure prediction approach, in this step the same concept to significantly larger test structures is applied. As seen in Figure 2-b, large scale pressure vessels of $2600 \mathrm{~mm}$ length and $890 \mathrm{~mm}$ diameter are fabricated using the filament winding process as described in the previous sections. 
The large-scale pressure vessel LPV-1 (outer dimensions similar to Figure 2-b) is designed to withstand an internal pressure of approximately 100 bar as maximum expected operational pressure (MEOP). The required minimum burst pressure shall be 1.25 times MEOP. The wall thickness is calculated not only to withstand the internal pressure but also to fulfill the required axial and radial deformations. LPV-1 is categorized as thin-walled pressure vessel with a maximum wall thickness $<5 \mathrm{~mm}$. It was fabricated without skirt and was loaded solely by internal pressurization.

The other large-scale pressure vessel, designated LPV-2, uses a skirt region (confirm Figure 2-b), which was fabricated in a combination of dry fiber placement and dry filament winding processes and subsequent resin infusion and curing. The scaling philosophy for LPV-2 is different compared to the approach followed for LPV-1. The wall thickness was in the range up to $25 \mathrm{~mm}$, which means that it is considered a thickwalled pressure vessel. The pressure vessel and skirt are joined together using adhesive bonding. In addition to the internal pressurization, the bottom part of the skirt is statically fixed against a metallic load frame using a double lap interface ring with two rows of bolts. Since the polar opening is mounted on a piston an axial load flux is induced into the skirt, which superimposes the internal (bi-axial) pressurization of the vessel (see Figure 3). While LPV-1 was equipped with 18 strain gages LPV-2 was equipped with 20 strain gages.

For both vessels, the test plan follows the three phases described in Section 3.1.

As seen in Figure 2-b, the selected $28 \mathrm{AE}$ sensor positions allow a $\mathrm{PoD} \geq 67 \%$ coverage applying the attenuation mapping approach proposed in [6]. In total, eight sensors are distributed across the bottom dome (hidden in Figure 2-b by skirt and pressure vessel) while the remaining 20 sensors are distributed on the cylinder and the upper dome. All acquisition settings are in accordance with the reference database, except for the trigger settings, which are chosen as 20/80/1500 $\mu$ s (Peak-DefinitionTime/Hit-Definition-Time/Hit-Lockout-Time) to account for the change in signal length due to guided wave dispersion at larger propagation distances. Similar to the SPV tests, all WD sensors are attached using custom build systems using adhesive bonding of the attachment frame to the vessels. For the attenuation mapping and the neural 
network based source localization procedure, a training grid of 272 (LPV-1) and 207 (LPV-2) (xyz)-locations was used. Similar to the SPVs, the obtained $\Delta t$-values are input to the training stage of an artificial neural network with two hidden layers and ten neurons each and are mapped against the corresponding (xyz)-locations. Due to the test fixture concept applied in the LPV-2 test, no Hsu-Nielsen test sources could be applied at the bottom dome at the time of test. Accordingly, source localization was carried out using classical $\Delta t$-based algorithms applying an isotropic sound velocity of $4500 \mathrm{~m} / \mathrm{s}$ on the bottom dome. This sound velocity is based on a measurement on the corresponding upper dome (same dimension and layup). Based on mutual pulsing of the sensor network, the average propagation velocity was determined and validated using localization of the Hsu-Nielsen test positions on the upper dome. The obtained $\mathrm{AE}$ source coordinates are merged with the results of the artificial neural network based source localization of the rest of the vessel. In summary, the approach presented in [11] allows to evaluate the mean source localization accuracies of $\leq 73 \mathrm{~mm}$ (LPV-1) and $\leq 104 \mathrm{~mm}(\mathrm{LPV}-2)$.

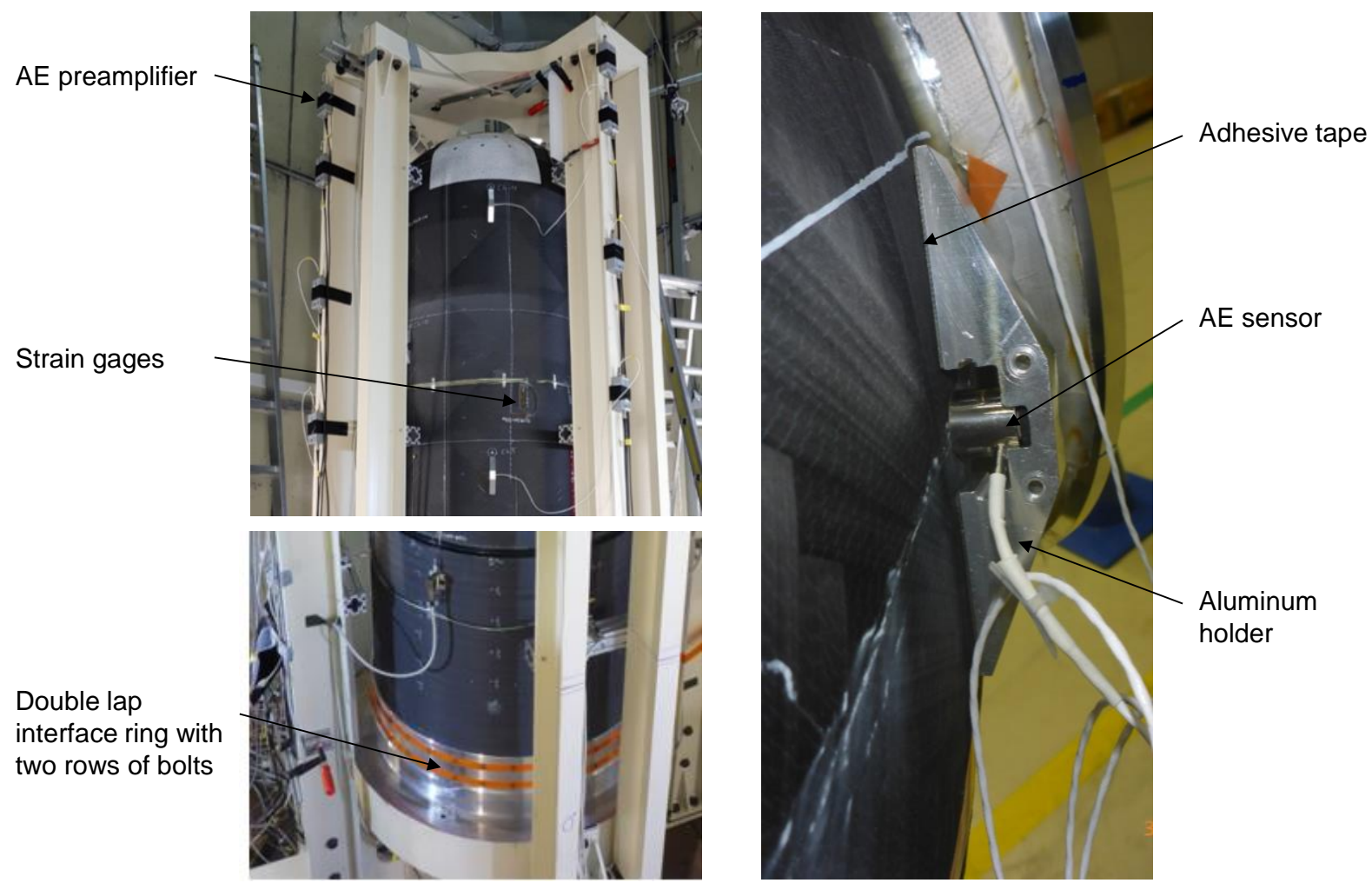

Figure 3: Details of LPV-2 load frame configuration (left) and AE sensor attachment (right). 
In order to confirm with the reference database one final step is required prior to the calculation of cyclic AE criteria. Due to the large distances of propagation in these tests, the $E R_{N}$ features are influenced significantly by attenuation. For localized $\mathrm{AE}$ signals, it is possible to apply a parameter correction technique to calculate the expected feature values without propagation influence. To this end, the source location information and attenuation mapping data is used to apply the technique proposed by Al-Jumaili et al. [8]. For global and local failure prediction, only localized AE sources and distance corrected features are taken into account.

\section{Results}

In this section, the results of the global and local failure prediction approaches are presented and compared to in situ observations by camera systems, strain gage monitoring and post mortem inspection of the vessels.

\subsection{Small-scale pressure vessels}

Based on the cyclic load schedule applied to the SPVs it is straightforward to calculate the $F R_{N}, S R_{N}$ and $E R_{N}$ ratios and to apply the corresponding $L R$ prediction for each cycle using Equation (5). Intentionally, the maximum peak load in phase two was planned for $80 \%$ of the expected burst pressure. However, SPV-1 and SPV-2 failed prematurely within the cyclic phase. Consistently, global forward prediction is based on $\mathrm{AE}$ data acquired for $L R<80 \%$, i.e. not all cycles of phase 2 are taken into account for SPV-1 and SPV-2. The forward prediction result of SPV-3 is shown as an example in Figure 4-a. The direct application of $f_{\text {opt }}$ trained based on the reference database is able to predict the load ratios of each cycle very well. In addition, in Figure 4-b the global burst pressure prediction results of all SPVs are compared to the measured values. For all cases, the deviation from the actual burst pressure is less than $3.0 \%$, with no more than $9.8 \%$ prediction uncertainty. 
(a)

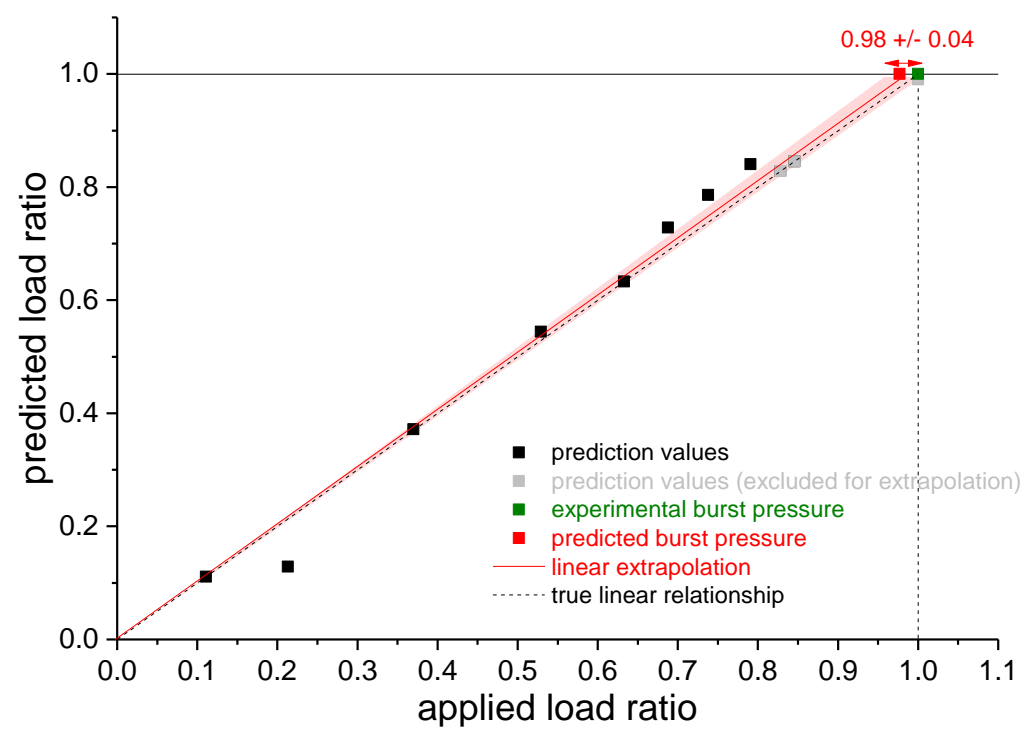

(b)

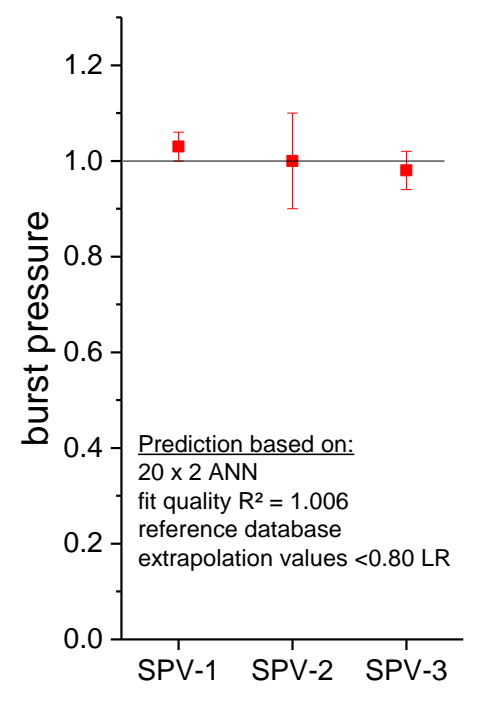

Figure 4: Example of forward extrapolation to obtain global failure prediction for SPV-3 (a) and comparison of global prediction values of SPV-1, SPV-2 and SPV-3 and respective measured burst pressure values (b), all normalized to unity for better comparison.

The artificial neural network based localization approach results in a source localization accuracy of $\leq 36 \mathrm{~mm}$ for SPV-3. Accordingly, the vessel is segmented in $i=5, j=5$ and $k=15$ sub-volumes to match the geometric dimension of the sub-volumes to this uncertainty of AE source positions. Based on the algorithm proposed in Section 2.2, a $L R$ prediction is made for each cycle in each sub-volume. The visualization in Figure 5 shows the predicted $L R$ color-coded to the localized signals inside each cube. As no smoothing is made across sub-volume borders, this causes the chessboard appearance seen in Figure 5. As an implicit limitation of this approach, $L R$ prediction is only possible for regions with localized $A E$ signals, since otherwise no data is available for the prediction procedure. However, it is unlikely that regions without $A E$ are highly prone to failure, since in composites this would typically result in generation of AE. Accordingly, all regions without color-code information are expected to exhibit a local $L R$ significantly less than the globally applied $L R$ (reported in Figure 5). Accordingly, the regions with predicted $L R$ significantly higher than globally applied are expected to face the highest stress exposure. As none of the SPVs is fabricated perfectly uniform and flawless, it is likely, that some regions exhibit local variations of fiber volume fractions, local fiber misalignment or other imperfections resulting from the filament winding process respectively from the chosen lay-up. For the SPV-3 this 
causes an early concentration of high $L R$ values in the upper equator region and the upper pole region. For SPV-3, the post mortem inspection supports the region indicated in Figure 5 as likely rupture positions. For this vessel, massive fragmentation reaching from pole to meridian of the vessel and with predominant radial position at the designated (xy)-position of highest $L R$ prediction was found after testing (see Figure 5). This rupture region correlates very well with the failure prediction and qualitatively with the strain gage readings. Comparisons of regions with high $L R$ prediction and post mortem analysis of SPV-1 and SPV-2 show similar results.

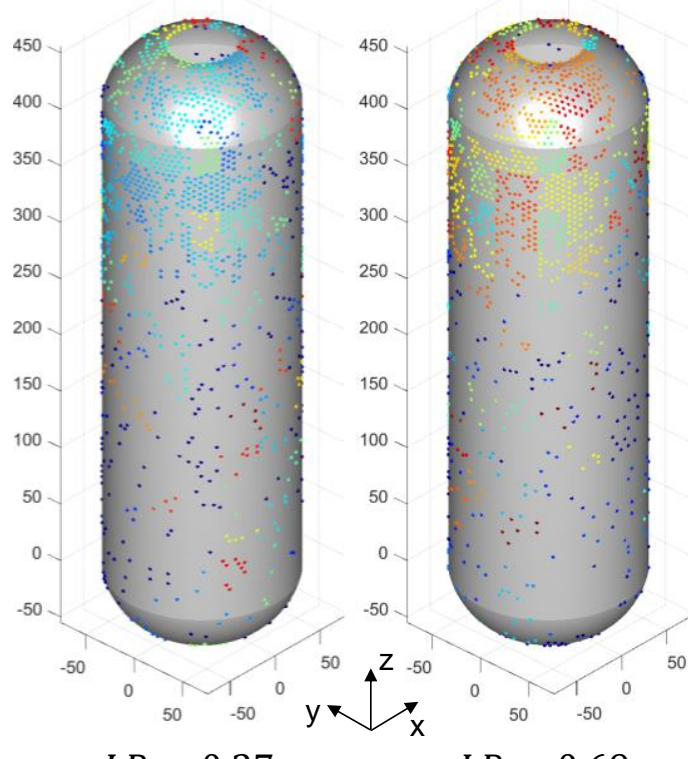

$L R=0.37$

$L R=0.69$

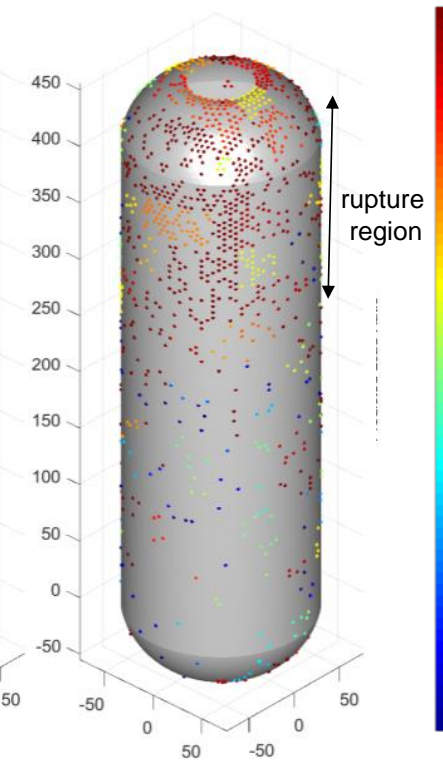

$L R=1.00$

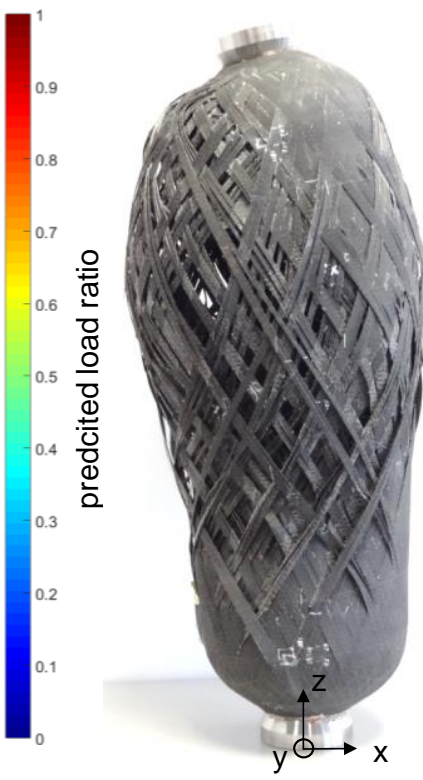

Figure 5: Example for local failure prediction of SPV-3, visualized as predicted $L R$ at cycle peak load in three subsequent cycles and post mortem image of SPV-3

\subsection{Large-scale pressure vessels}

Following the same approach as discussed in section 4.1, the $L R$ prediction to the test cases of LPV-1 and LPV-2 is applied. For these test cases, the forward prediction is based on $A E$ data acquired for $L R<72 \%$ in the case of LPV-1 and $L R<53 \%$ in the case of LPV-2. The forward extrapolation to predict the burst pressure is shown in Figure 6-a for LPV-2. The global burst pressure is predicted within the margin of error for both cases as seen in Figure 6-b. This demonstrates that the database obtained on lab-scale specimens is feasible to capture the significant change in length scale between SPV and LPV. This is only possible as the AE criteria defined in section 2, 
are independent of the test volume and the energy release as they are normalized by the corresponding values of the previous cycle. As pointed out in [15], their values should therefore not depend on the tested volume or AE activity. The latter is only true as long as independent (transient) signals are acquired and no continuous $A E$ is observed. Moreover, the applied parameter correction technique (see Al-Jumaili et al. [8]) guarantees an equal interpretation of energetic $A E$ features, which is required for this application of $L R$ prediction. For LPV-1, the deviation from the actual burst pressure is only $2.4 \%$, with $6.0 \%$ prediction uncertainty. For LPV-2 the deviation is $3.0 \%$, with $9.4 \%$ prediction uncertainty.

(a)

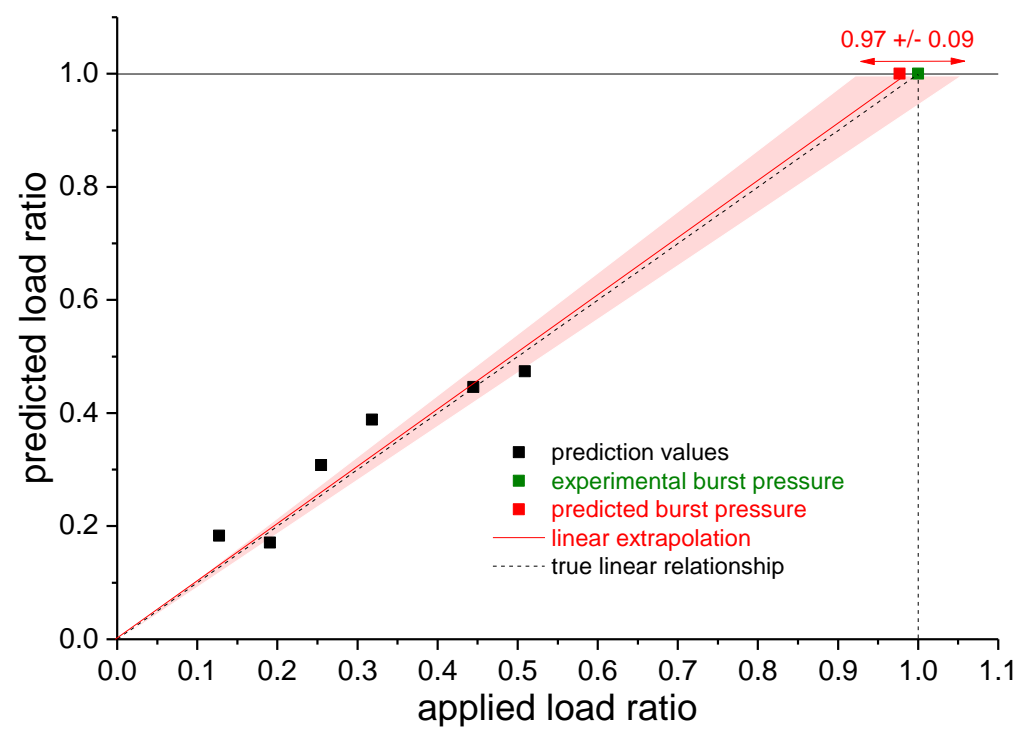

(b)

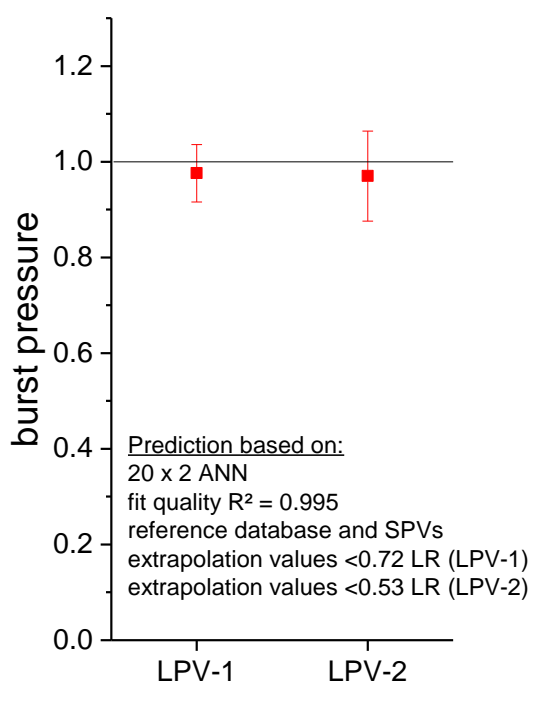

Figure 6: Example of forward extrapolation to obtain global burst pressure prediction for LPV-2 (a) and comparison of global prediction values for LPV-1 and LPV-2 and measured burst pressure values (b), all normalized to unity for better comparison.

For both LPV cases, a significant number of AE signals $\left(>10^{6}\right)$ were detected and localized during the test. Similar to the SPV cases, based on the vessel dimensions and the source localization accuracies of $73 \mathrm{~mm}$ and $104 \mathrm{~mm}$, sub-volumes of $i=10$, $j=10$ and $k=29$ are chosen. Examples of the predicted $L R$ are shown in Figure 7 for four different global $L R$ values of the LPV-1 test. The style of presentation follows the description of Section 4.1. As function of the global $L R$ values, there is a similar trend in the mean values calculated locally. AE sources are predominantly occurring in the upper part of the cylindrical region and in the area of the lower dome and equator. 
Starting at $L R \geq 0.53$, some regions in the lower part of the vessel start to exceed the global load ratios significantly and approach local values of $L R \approx 1$. This is indicative of imminent structural damage at these spots (e.g. rupture of fiber strands), which was audible at these load levels during the pressurization. Other than classically expected, both dome regions are not loaded symmetrically, e.g. much more damage is concentrated in the lower dome region. With further increase of pressure, more regions in the lower dome and in the upper cylindrical part show very high $L R$ values until burst rupture of LPV-1 occurs at the bottom dome. This initial failure spot was confirmed by simultaneous high-speed camera monitoring (see image sequence in Figure 7 starting at moment of burst $\left.t_{1}\right)$. Nevertheless, huge portions of the cylindrical part are exhibiting very high $L R$ values, indicating, that these were imminent to fail as well.
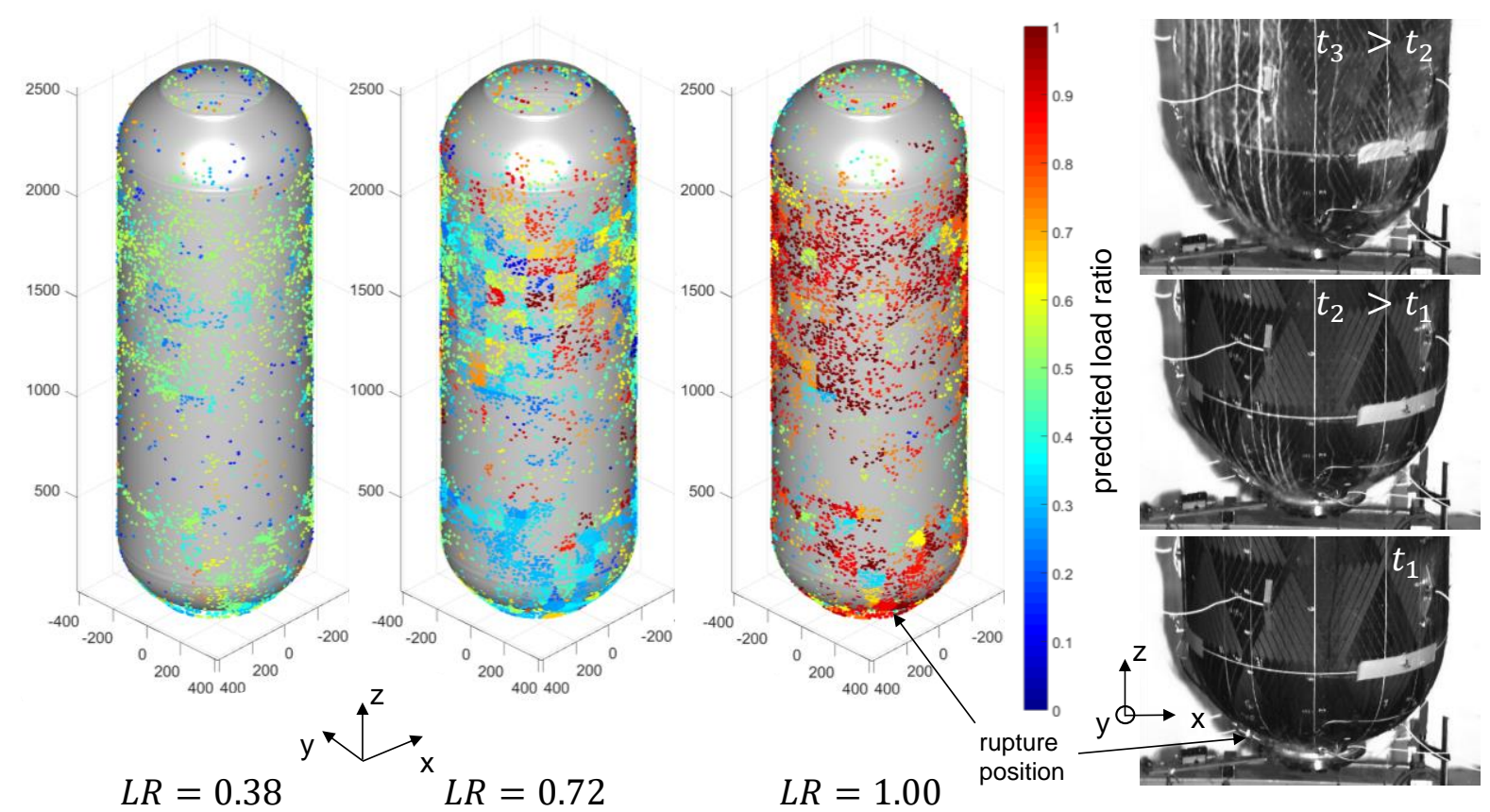

Figure 7: Example for local failure prediction of LPV-1, visualized as predicted $L R$ at cycle peak load in four subsequent cycles.

For the LPV-2 example shown in Figure 8, the predicted local $L R$ values appear much more homogeneous than for the SPV-3 in Figure 5 and the LPV-1 in Figure 7. As these cyclic tests were carried out only up to $L R=0.53$ this is shown as final cycle. In this image, the local $L R$ values are fairly homogenous and little exceedance of the global $L R$ value is observed. The local $L R$ values are also in good agreement with the global 
$L R$ value. For lower $L R$ values, some local values up to $L R=0.93$ are observed. Similar as discussed for the LPV-1 case, this likely resulted in some significant local damage that does not cause immediate rupture of the vessel. Overall, this indicates a uniform stress state of the pressure vessel. In general, this impression can be confirmed qualitatively by the evaluation of the strain measurements. However, the strains measured in the dome regions are somewhat lower than the strains measured in the cylinder, the overlapping area of skirt and pressure vessel and at the bulges around the polar openings. This effect can be explained with the winding pattern, which leads to a lower filament utilization in the dome region. The post mortem analysis of LPV-2 is in accordance with this fairly uniform stress state during pressurization. Due to the absence of a video documentation the post mortem analysis does not allow identifying a definite failure initiation location. However, since two halves of the cylinder, a huge part of the igniter dome and the nozzle dome with a partly destroyed skirt were remaining, a rupture in the igniter dome close to the equator or a rupture of the cylinder are likely.

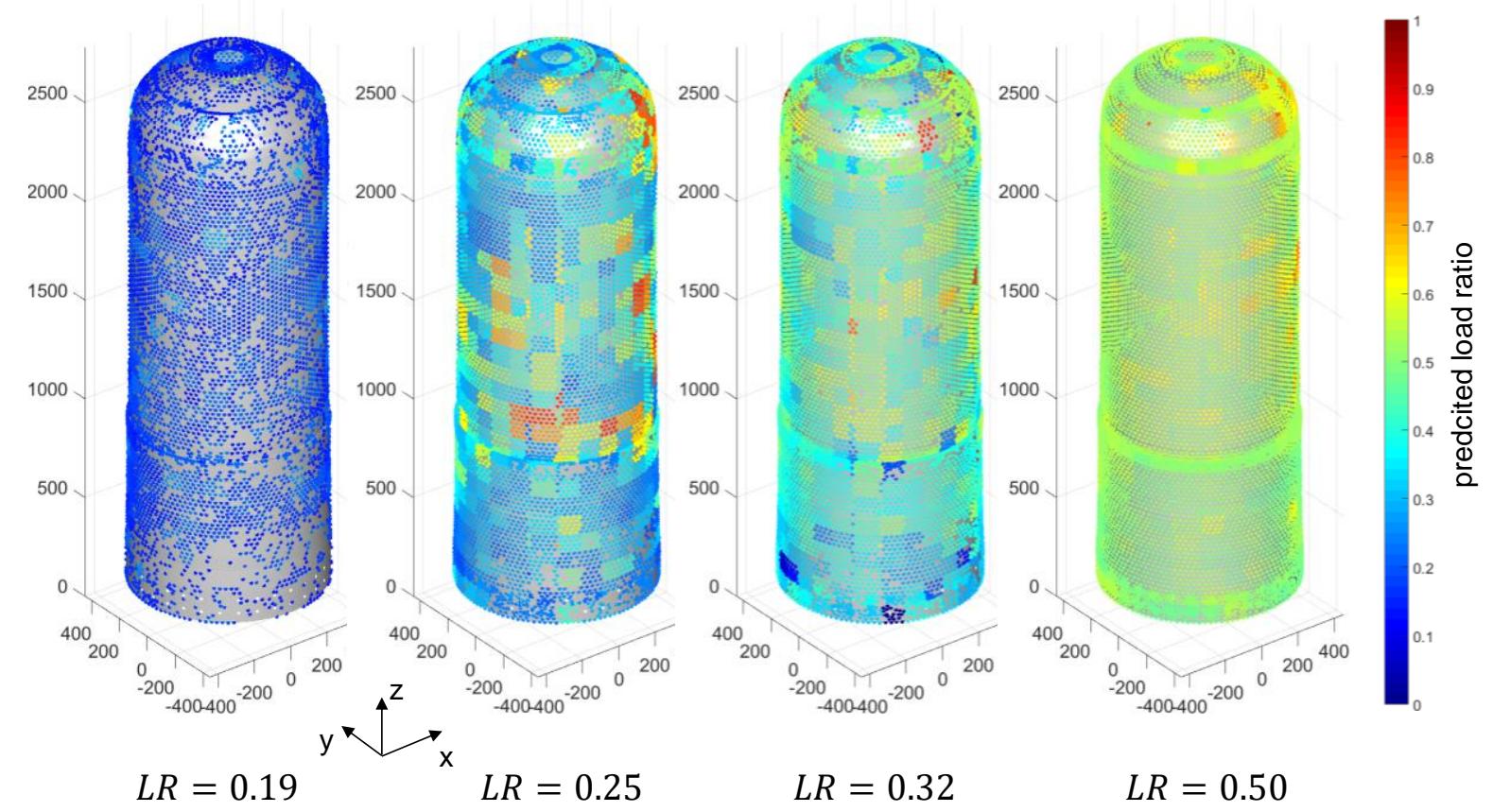

Figure 8: Example for local failure prediction of LPV-2, visualized as predicted $L R$ at cycle peak load in four subsequent cycles. 


\section{Conclusion}

A novel approach to predict local load ratios of fiber reinforced structures has been proposed. The prediction on structural scale is using a reference database established throughout typical lab-scale experiments for material qualification. In combination with artificial neural network based source localization and parameter correction techniques, this proofs sufficient to predict the global burst pressure of ten times larger pressure vessels. In addition, the sub-volume segmentation presented herein allows prediction of local load ratios for each load cycle. Comparison was made between regions of predicted high load ratios, in situ camera observations and post mortem analysis. Based on the good agreement in all five test cases analyzed, this allows to conclude, that such load ratio prediction can identify regions with high stress exposure, which are most likely the positions of failure initiation and ultimately global failure. The provided approach helps to understand the structural behavior of the pressure vessels and leads to a better understanding of the involved failure mechanisms. Moreover, with its predictive capabilities the local load ratio can directly be interpreted as measure of stress exposure and may act as useful experimental reference for predictions based on numerical analysis techniques such as finite element modeling. Currently, this is already used to advance numerical calculation methods to describe damage progression in pressure vessels. Moreover, the use of this AE approach may provide sufficient feedback from single design iterations such as winding patterns to avoid testing an excessive number of test items. Another future prospective of the global prediction approach is the use within quality control, e.g. during pressure vessel qualification or during re-certification.

\section{Acknowledgments}

We would like to thank the European Space Agency ESA for funding the development project "P120C $2^{\text {nd }}$ Production Line Step 1a Technology Demonstration" in which the presented results have been obtained.

\section{Literature}

[1] A.S. Kaddour, M.J.M.J. Hinton, Maturity of 3D failure criteria for fibre-reinforced 
composites: Comparison between theories and experiments: Part B of WWFEII, J. Compos. Mater. 47 (2013) 925-966. doi:10.1177/0021998313478710.

[2] P.D. Soden, a. S. Kaddour, M.J. Hinton, Recommendations for designers and researchers resulting from the world-wide failure exercise, Compos. Sci. Technol. 64 (2004) 589-604. doi:10.1016/S0266-3538(03)00228-8.

[3] A.S. Kaddour, M.J. Hinton, P.D. Soden, A comparison of the predictive capabilities of current failure theories for composite laminates: Additional contributions, Compos. Sci. Technol. 64 (2004) 449-476. doi:10.1016/S02663538(03)00226-4.

[4] M.G.R. Sause, In Situ Monitoring of Fiber-Reinforced Composites, Springer International Publishing, Cham, 2016. doi:10.1007/978-3-319-30954-5.

[5] M. Sause, M. Hamstad, Acoustic Emission Analysis, in: P.W.R. Beaumont, C.H. Zweben (Eds.), Compr. Compos. Mater. II, Elsevier, Oxford, 2018: pp. 291-326. doi:10.1016/B978-0-12-803581-8.10036-0.

[6] M.G.R. Sause, F.F. Linscheid, M. Wiehler, An Experimentally Accessible Probability of Detection Model for Acoustic Emission Measurements, J. Nondestruct. Eval. 37 (2018) 17. doi:10.1007/s10921-018-0474-4.

[7] A. Pollock, Probability of Detection for Acoustic Emission, 25 (2007) 167-172.

[8] S.K. Al-Jumaili, K.M. Holford, M.J. Eaton, R. Pullin, Parameter Correction Technique (PCT): A novel method for acoustic emission characterisation in large-scale composites, Compos. Part B Eng. 75 (2015) 336-344. doi:10.1016/j.compositesb.2015.01.044.

[9] E. Maillet, N. Godin, M.R. Mili, P. Reynaud, G. Fantozzi, Lifetime prediction with acoustic emission during static fatigue tests on ceramic matrix composite at intermediate temperature under air, (2014) 22-26.

[10] C.U. Grosse, M. Ohtsu, Acoustic Emission Testing in Engineering - Basics and Applications., Springer, Berlin, Heidelberg, 2008. doi:10.1007/978-3-54069972-9.

[11] S. Kalafat, M.G.R.G.R. Sause, Acoustic emission source localization by artificial neural networks, Struct. Heal. Monit. 14 (2015) 633-647. doi:10.1177/1475921715607408.

[12] S.K. Al-Jumaili, M.R. Pearson, K.M. Holford, M.J. Eaton, R. Pullin, Acoustic emission source location in complex structures using full automatic delta $T$ mapping technique, Mech. Syst. Signal Process. 72-73 (2016) 513-524. doi:10.1016/j.ymssp.2015.11.026.

[13] M.J. Eaton, R. Pullin, K.M. Holford, Acoustic emission source location in composite materials using Delta T Mapping, Compos. Part A Appl. Sci. Manuf. 43 (2012) 856-863. doi:10.1016/j.compositesa.2012.01.023.

[14] K.S. Downs, M.A. Hamstad, Acoustic Emission from Depressurization to Detect/Evaluate Significance of Impact Damage to Graphite/Epoxy Pressure Vessels, J. Compos. Mater. 32 (1998) 258-307. doi:10.1177/002199839803200304.

[15] M.G.R. Sause, S. Schmitt, S. Kalafat, Failure load prediction for fiber-reinforced composites based on acoustic emission, Compos. Sci. Technol. 164 (2018) 24-33. doi:10.1016/j.compscitech.2018.04.033.

[16] K.S. Downs, M.A. Hamstad, Correlation of Acoustic Emission Felicity Ratios and Hold-Based Rate Moments with Burst Strngths of Spehrical Graphite/Epoxy Pressure Vessels, J. Acoust. Emiss. 13 (1995) 45-56.

[17] T.J. Fowler, Revisions to the CARP Recommended Practice for Tanks and Vessels, in: Proc. Fifth Int. Symp. Acoust. Emiss. From Compos. Mater. AECM-5, American Society for Nodestructive Testing, Columbia, Ohio, 
Sundsvall, Sweden, 1995: pp. 263-271.

[18] T.J. Fowler, Acoustic Emission Testing of Fiber Reinforced Plastics, Proc. Pap. J. Tech. Counc. ASCE. 105(TC2) (1977) 281-289.

[19] J.M. Waller, C.T. Nichols, D.J. Wentzel, R.L. Saulsberry, D.O. Thompson, D.E. Chimenti, Use of Modal Acoustic Emission to Monitor Damage Progression in Carbon FiberEpoxy Composites, in: AIP Conf. Proc., San Diego, California, (USA), 2011: pp. 919-926. doi:10.1063/1.3592036.

[20] E.K. Hill, S.T. Dion, J.O. Karl, N.S. Spivey, J.L.W. li, Neural Network Burst Pressure Prediction in Composite Overwrapped Pressure Vessels, Neural Networks. 25 (2007) 187-193.

[21] J.L. Walker, E.V.K. Hill, Backpropagation neural networks for predicting ultimate strengths of unidirectional graphite/epoxy tensile specimens, Adv. Perform. Mater. 3 (1996) 75-83. doi:10.1007/BF00136861.

[22] J.L. Walker, G.L. Workman, S.S. Russell, E.V.K. Hill, Neural network/acoustic emission burst pressure prediction for impact damaged composite pressure vessels, Mater. Eval. 55 (1997). http://www.osti.gov/scitech/biblio/562042 (accessed May 2, 2015).

[23] S. Rajendraboopathy, T. Sasikumar, K.M. Usha, E.S. Vasudev, Artificial neural network a tool for predicting failure strength of composite tensile coupons using acoustic emission technique, Int. J. Adv. Manuf. Technol. 44 (2009) 399-404. doi:10.1007/s00170-008-1874-x.

[24] H.M. Deuschle, A. Puck, Application of the Puck failure theory for fibrereinforced composites under three-dimensional stress: Comparison with experimental results, J. Compos. Mater. 47 (2013) 827-846. doi:10.1177/0021998312462158.

[25] A. Puck, Failure analysis of FRP laminates by means of physically based phenomenological models, Compos. Sci. Technol. 58 (1998) 1045-1067. doi:10.1016/S0266-3538(96)00140-6. 